\title{
Energy-efficient Relay Selection Schemes for Contention-based Geographic Forwarding
}

\author{
Ibrahim Amadou \\ Université de Lyon \\ INRIA / INSA-Lyon, CITI \\ F-69621 Villeurbanne, FR. \\ ibrahim.amadou@insa-lyon.fr
}

\author{
Anis Ouni \\ Université de Lyon \\ INRIA / INSA-Lyon, CITI \\ F-69621 Villeurbanne, FR. \\ anis.ouni@insa-lyon.fr
}

\author{
Hervé Rivano \\ Université de Lyon \\ INRIA / INSA-Lyon, CITI \\ F-69621 Villeurbanne, FR. \\ herve.rivano@inria.fr
}

\author{
Fabrice Valois \\ Université de Lyon \\ INRIA / INSA-Lyon, CITI \\ F-69621 Villeurbanne, FR. \\ fabrice.valois@insa-lyon.fr
}

\begin{abstract}
Contention-based Geographic Forwarding (CGF) has emerged recently as an energy-efficient communication protocol to improve the wireless sensor networks lifetime. By exploiting the wireless broadcast advantage and node spatial diversity, it mitigates the effect of varying channel condition and node unavailability. The main contributions are based on classical relay selection scheme such as most forward within radius. However, this relay selection scheme performs poorly under real radio environments because it tends to forward packets on unreliable link, which leads to high expected number of retransmissions in each hop and an important number of duplicated packets. In this paper, we investigate the performance of several relay selection schemes. First, extensive simulations are proposed to evaluate their performance locally in terms of packet delivery ratio and duplicated packets. Then, we extend the work in multihop wireless networks and evaluate their performance in term of energy. To highlight their gain, we propose a smart solution obtained by linear programming. Based on the intelligent relay selection scheme, we develop a new solution, Furthest Forward within Reliable neighbors $\left(F F R_{e}\right)$, which tries to save more energy than the most efficient relay selection schemes.
\end{abstract}

\section{INTRODUCTION}

Contention-based Geographic Forwarding protocols (CGF) were firstly developed to eliminate the proactive beaconing drawbacks of geographic routing protocols in wireless sensor networks (WSNs). Unlike the well known geographic routing such as GPSR [11], they do not use pre-requisite knowledge of the neighborhood. Local informations are known reactively through distributed contention process. Basically, the core idea of CGF routing protocols is to combine greedy forwarding with recovery forwarding. In greedy forwarding, packets are broadcast in the neighborhood and then, the next hop is selected distributively inside a dedicated area called forwarding area by a contention scheme using an additional delay. This delay is computed by each node according to the expected forward progress or distance or remaining energy. Recovery forwarding is used when there is no forwarder candidate (hole problem [3]). The difference among the existing CGF protocols comes from either the relay selection scheme (priority policy [17]) or the distribution contention function or the forwarding area design or the recovery strategy. Prior studies mainly focus on networks with high density in order to perform in greedy mode, assume an ideal disk channel model ${ }^{1}$

${ }^{1}$ Radio links are perfect within a given communication range and focus on finding the shortest path from a source to a destination. However, while assuming high density network deployment may be acceptable in wireless sensor networks, it is now clear that the perfect radio links assumption is unlikely to be valid with realistic physical layer model. In fact, wireless links are more unstable due to varying link qualities and unreliable than wired networks. These behaviors lead to poor performance when explicit neighbor discovery is used during the forwarding process, a large number of retransmissions and high duplicated packets, which can increase network congestion.

In this paper, we investigate the performance in terms of packet delivery ratio and duplicated packets of the existing relay selection schemes for CGF or geographic routing protocols under a realistic wireless channel model and considering only the 1-hop neighborhood. Then, we focus on multi-hop wireless networks using these previous CGF forwarding strategies. To highlight the performance in term of energy consumption. Results are compared with an optimal solution given by a linear programming. Based on these studies, we develop a new solution Furthest Forward within Reliable neighbors $\left(F F R_{e}\right)$, which tries to minimize both the per-hop expected number of retransmissions and the number of hops in order to save energy.

The rest of the paper is organized as follows: Section II presents some existing relay selection schemes and some well known proposed relay selection schemes under realistic wireless channel model. Section III introduces our assumption and our radio channel model. Section IV presents a performance evaluation by simulation while the section $\mathrm{V}$ presents analytically the extension in case of multi-hop wireless networks. Analytical results are presented in Section VI. Section VII presents our proposal relay selection scheme and evaluate its gain. Section VIII concludes this work.

\section{RELATED WORK}

Unlike the recent works such as [4], [10], [16]-[18], that try to improve the existing routing protocol relay selection policies in order to take into account the realistic wireless channel model or to improve the network capacity or the energy efficiency by keeping the shortest path metric approach, in this work, our aim is to study both the reliability and 
energy efficiency of the different relay selection schemes for greedy geographic forwarding. Most Forward within Radius (MFR)/Most Forwarding Progress (MFP) [15], Nearest Forwarding Progress (NFP)/Nearest Forward within Radius (NFR) [7] and Random Progress Method (RMP) [13] are some of the earliest position-based routing protocol that were proposed for packet radio networks and were lately adopted for wireless ad hoc and sensor networks such as GPSR [11] or BLR [2]. When MFR/MFP is used, at each hop, the node forwards its packets to the neighbor that maximizes the euclidean distance or the progress ${ }^{2}$. NFR/NFP was proposed in order to maximize throughput in wireless networks by selecting its relay node among the nearest neighbors in terms of the euclidean distance or the progress. While RMP was proposed in order to introduce a load balance during the forwarding. Compass or Angular [12] was proposed in 1999s for ad hoc networks. Angular forwarding consists to forward the packets to the neighbor that has the smallest angle. It means that the selected forwarder is the neighbor which has lower angle between the destination node, the source node and itself.

\section{Assumptions AND RADIO MODEL}

In this section, we present the assumptions and radio link model used in this work.

\section{A. Assumptions}

We consider a WSN composed of sensor nodes. The nodes are randomly deployed with uniform distribution on 2D plane. We assume that sensors are aware of their own locations and know the location and the identity of sink node in the plane. There is no assumption about the properties of radio channel: channel may be either symmetric or asymmetric depending on radio environment. Radio communication range is unknown parameter, it depends on transmission power of each sensor and also of the radio environment. Given the wireless broadcast advantage, a packet sent by a node is assumed to be received by its neighbors. Packets are assumed to be acknowledged hop by hop either explicitly as in [?] or implicitly as in [6]. Finally, we consider that there is no hole in the network in order to guarantee the use of greedy forwarding strategy.

\section{B. Radio channel model}

For both simulation and analysis undertaken in this study, we consider a lognormal shadowing model, which is useful when dealing with more realistic radio environment. In our analysis, as in [9] and based on DSSS-OQPSK modulation of CC2420 chip for MicaZ nodes, the probability of successfully receiving packet with length $l$ bits can be expressed as :

$$
p_{f}(\gamma(d))=\left(1-Q\left(\sqrt{2 \frac{B}{R_{b i t}} 10^{\frac{\gamma(d)}{10}}}\right)\right)^{l}
$$

\footnotetext{
${ }^{2}$ it is defined as the projection of the distance traveled over the last hop from the forwarder to the final destination $\mathrm{D}$
}

Where $\gamma(d)=P_{t}-P L\left(d_{0}\right)-10 \eta \log \left(\frac{d}{d_{0}}\right)+X_{\sigma}-P_{n}$ is signal-to-noise ratio at the transmitter-receiver distance $d$. $P_{t}, P L\left(d_{0}\right), X_{\sigma}$ and $P_{n}$ are respectively the transmission power in $\mathrm{dBm}$, the path loss at a reference distance $d_{0}$ in $\mathrm{dBm}$, the shadow fading component, with $X_{\sigma} \sim \mathcal{N}(0, \sigma)$ and the noise power in $\mathrm{dBm}$. The $Q($.$) is the Q-function.$ $R_{b i t}$ is the radio bit rate while $B$ is the noise bandwidth. Let us now define $p_{r}(\gamma(d))$ as probability of successfully receiving acknowledgment from receiver after the transmission of data packet. This acknowledgment can be either a data packet or an explicit acknowledgement packet $A C K$. We define $p_{u}=p_{f}(\gamma(d)) \times p_{r}(\gamma(d))$ as the probability of successful and acknowledged transmission in 1-hop. This means that both the unicast data and acknowledgment packets are successfully received. Let us consider the overall probability $p$ follows a geometrical distribution with parameter $p_{u}$, which is given by:

$$
p=\left[\prod_{j=1}^{i-1}\left(1-p_{u}\right)\right] \times p_{u} .
$$

The expected number of retransmissions is then defined by $N_{T x}=\sum_{i=1}^{\infty} i \times p$ and can be expressed as:

$$
N_{T x}=\sum_{i=1}^{\infty} i \times p_{u} \times\left(1-p_{u}\right)^{i-1}=\frac{1}{p_{u}}
$$

\section{RELAY SELECTION SCHEMES IN 1-HOP NEIGHBORHOOD}

The purpose of this study is to evaluate the effect of realistic radio model on performance of relay selection schemes in the 1-hop neighborhood of the source node. We extensively evaluate the performance of relay selection schemes through WSNET [1] simulations. We evaluated their performance according to the following metrics:

- Average Packet Delivery Ratio: It defines the average ratio of total number of packets successfully relayed by the next hop in the neighborhood of the source node to total number of packets generated by the source node. Packet is assumed to be successfully relayed if and only if the source node is aware of its relaying. It determines the reliability of relay selection scheme. More reliable is the relay selection scheme, better its performance is in terms of the number of duplicated packets and network congestion. It is useful when dealing with asymmetric links.

- Average Duplicated packet: It provides the average number of times the packet is relayed after its first retransmission.

The results are presented with a confidence interval of $95 \%$. Simulation parameters are summarized in the table I.

\section{Results and discussions}

Fig. 1 and Fig. 2 display the average packet delivery ratio and average number of duplicated packets of the six reviewed relay selection schemes when the source node degree increases. From the two figures, we can make out that whatever the 


\begin{tabular}{|l|l|}
\hline Radio bit rate & 250Kbps \\
\hline Path Loss exponent $\eta$ & 3.75 \\
\hline Shadow fading variance $\sigma$ & 4 \\
\hline Transmission power $P_{t}$ & $0 \mathrm{dBm}$ \\
\hline Noise power $P_{n}$ & $-110 \mathrm{dBm}$ \\
\hline Antenna sensitivity & $-94 \mathrm{dBm}$ \\
\hline Data packet length & 112 bytes \\
\hline Source node degree & $10-24$ \\
\hline
\end{tabular}

TABLE I: Simulation parameters

degree, NFR is the most suitable relay selection scheme in terms of packet delivery ratio and the least number of duplicated packets. The reason is due to the use of the reliable short wireless links while the remaining other relay selection schemes select their next hop among nodes that have the unreliable long wireless links. This is due to the fact that at each hop they attempt to minimize the expected number of hops by maximizing the forwarding distance. The difference between NFR and NFP is mainly due to the fact that NFP uses the progress, which is the product of the distance with the cosinus of angle instead of the distance used by NFR. When the cosinus is very low, whatever the distance, their product is low. Therefore, NFP selects its next hop among nodes that present a lower cosinus of the angle and longer distance. This is likely to lead a significant performance degradation when the degree increases. This explains the difference between these two nearest forwarding schemes. The opposite reasoning can be applied to MFP and MFR when the progress is used instead of the distance. It explains their difference. While Angular and RMP present the similar performance with a small difference in term of the number of duplicated packets. This can be mainly explained through the similarity between their relay nodes distribution in the vinicity of the source node.

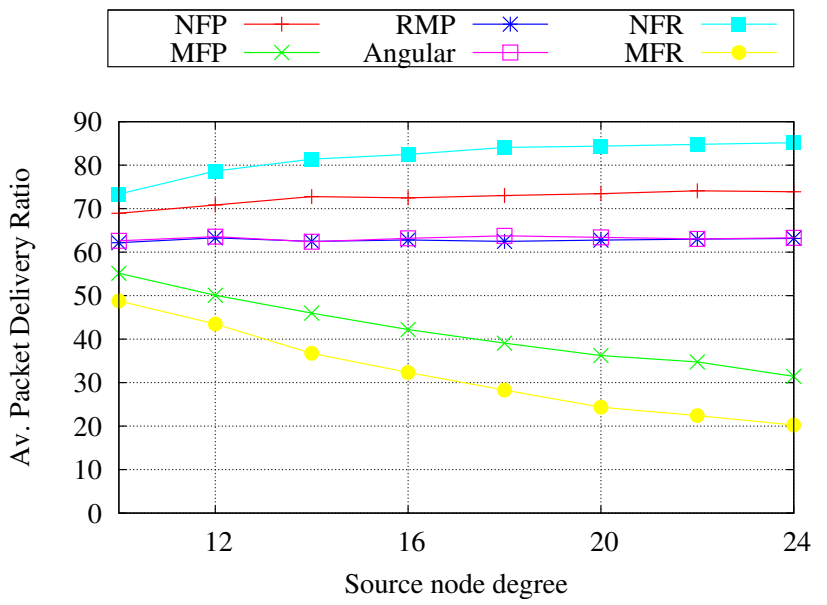

Fig. 1: Average packet delivery ratio vs Source node degree.

\section{OPTIMAL MULTI-HOP ROUTING TO SOLVE THE RETRANSMISSION PROBLEM}

In this section, we propose a linear program in order to compute an optimal routing solution which minimizes the

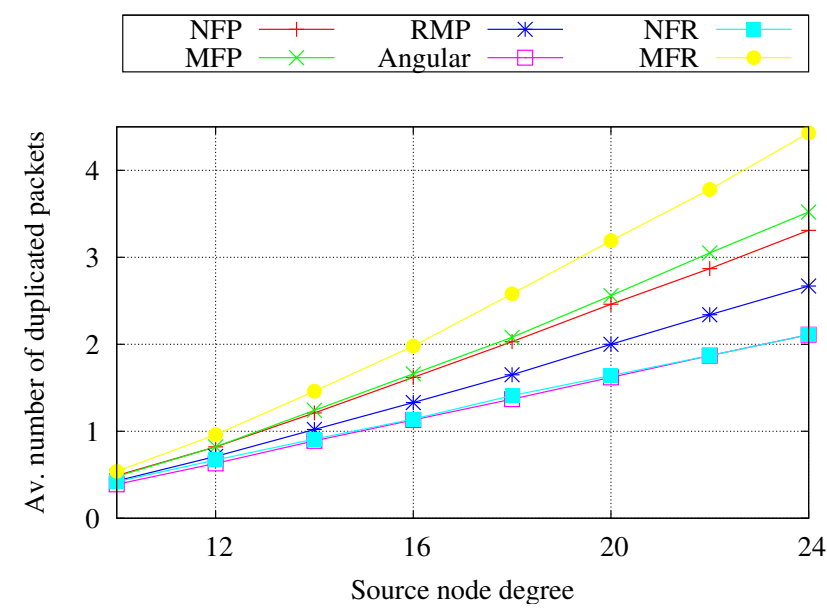

Fig. 2: Average number of duplicate packets vs Source node degree.

maximum energy consumption for sensor nodes.

\section{A. Network model and notations}

The static wireless sensor network is modeled as a directed graph $G(V, E)$, where $V$ and $E$ are respectively the set of nodes and links. The set of nodes $V$ is decomposed into one sink and a set of sensors that are respectively denoted by $S$ and $\mathrm{V}_{\mathrm{s}}$. Given a link $(\mathrm{u}, \mathrm{v}) \in \mathrm{E}, \mathrm{u}$ is the sending node and $\mathrm{v}$ is the receiving node. Each link is characterized by the capacity $C(u, v)$ and expected number of retransmissions $N_{r}(u, v)$. Both the link capacity and expected number of retransmissions depend on the modulation and coding scheme. The expected number of retransmissions expresses the radio link properties, where link with smaller expected number of retransmissions has higher link quality or is a reliable link (more details are presented in subsection III-B).

\section{B. Energy consumption model}

An important observation in the case of wireless sensor network is that the radio is the sensor node's component, which causes a significant energy dissipation [14]. In this work, we consider an energy consumption model based on the node operation modes: transmission, reception and idle. A node $u$ in transmission mode spends a transmitting cost $E_{t}(u)$, while each node $v$ able to receive the packet $((u, v) \in E)$, spends a receiving cost $E_{r}(v)$. We assume that each node consumes a fixed cost which corresponds to the idle state. This energy consumption model is described in Eq.(4). $f(r, u, v)$ is a binary variable which presents an unit of flow generated by the sensor $r$ and routed through link $(u, v)$.

$$
\begin{aligned}
\forall u \in V & \quad E(u)=\sum_{r \in V_{s}}\left(\sum_{v \in \Gamma_{+}(u)} N_{r}(u, v) * E_{t}(u) * f(r, u, v)\right. \\
+ & \left.\sum_{v \in \Gamma_{-}(u)} \sum_{w \in \Gamma_{+}(v)} N_{r}(v, w) * E_{r}(u) * f(r, v, w)\right)
\end{aligned}
$$




\section{Routing problem}

Each sensor $u \in V_{s}$ can generate and inject a quantity of traffic $d(u)$ into the network. This traffic is routed from the source node to the sink $S$ through several hops without loss. This route is calculated based on Eq.(5), which presents the conservation flow constraint. It guarantees that the unit of flow $f$ generated by a source node and entering a relay node is equal to the amount of its outflow. It also guarantees that the flow exiting at the source node is equal to the flow entering at the destination node. The link capacity constraints (6) impose that the total flow on the link $(u, v)$ does not exceed its capacity.

$$
\begin{aligned}
& \forall r \in V_{s}, v \in V \\
& \sum_{(v, w)} f(r, v, w)-\sum_{(w, v)} f(r, w, v)= \begin{cases}d(r), & \text { if } v=r \\
-d(r), & \text { if } v=S \\
0 & \text { if } v \in V_{s} \backslash\{r\}\end{cases} \\
& \forall(u, v) \in E \quad \sum_{r \in V_{s}} f(r, u, v) \leq C(u, v)
\end{aligned}
$$

\section{Linear programming formulation}

The life time of a sensor is mainly related to its total energy consumption during its operation. Hence, minimizing the energy consumption of sensors allows to maximize the life time of each sensor in the network. Our linear program computes the optimal route that minimizes the maximum consumption energy of all sensors, i.e., $\min _{\max } \operatorname{meV}_{s}\{E(u)\}$. This is a min-max optimization problem, respecting a number of constraints: flow conservation, capacity constraints and energy model consumption (Eq.(4)-Eq.(6)). We can then formulate this problem as follows:

$$
\begin{gathered}
\min _{f, E} \max _{u \in V_{s}} E(u) \\
\text { subject to Eq. (4)-Eq. (6) }
\end{gathered}
$$

Note that this linear program can calculate several routes which give the same optimal solution (min-max value). An optimal route is the one that contains the "min-max" consumption node that consumes more than each other nodes involved in the route. Hence, various routes with different number of hops are possible. To solve this problem, we choose among all routes the one that minimizes the total energy consumption $\left(\min \sum_{u} E(u)\right)$.

Table II summarizes all the linear programming parameters and notations.

\section{ANALYTICAL RESUlTS AND DISCUSSION}

In this section, we extensively evaluate the energy consumption of these relay selection schemes in the case of the multihop wireless sensor network.

\begin{tabular}{|c|c|}
\hline$E, V$ & Set of links and nodes \\
\hline$(u, v)$ & Radio link \\
\hline$C(u, v)$ & Capacity of the link $(u, v)$ \\
\hline$f(r, u, v)$ & $\begin{array}{c}\text { Flow of sensor } r \text { and routed } \\
\text { through link }(u, v)\end{array}$ \\
\hline$E_{t}(u)$ & $\begin{array}{c}\text { Energy consumption } \\
\text { of transmitter } u\end{array}$ \\
\hline$E_{r}(v)$ & $\begin{array}{c}\text { Energy consumption } \\
\text { of receiver } v\end{array}$ \\
\hline$\left.E_{(} u\right)$ & $\begin{array}{c}\text { Total Energy consumption } \\
\text { of node } u\end{array}$ \\
\hline$N_{r}(u, v)$ & $\begin{array}{c}\text { Expected number } \\
\text { of retransmissions of link }(u, v)\end{array}$ \\
\hline$d(u)$ & Traffic generated by node $u$ \\
\hline$\Gamma_{+}(u) / \Gamma_{-}(u)$ & $\begin{array}{c}\text { Set of outgoing/ingoing } \\
\text { links of node } u\end{array}$ \\
\hline
\end{tabular}

TABLE II: LP Model Notations

\section{A. Scenarios and Model Parameters}

We have developed and tested the linear programming formulation using AMPL/CPLEX [5], [8], to evaluate the performance in term of energy consumption of the different relay selection schemes. We consider a sensor network where 100 nodes are randomly deployed with uniform distribution on a network of dimension $100 \mathrm{~m} * 100 \mathrm{~m}$. For each pair of nodes $u$ and $v$, when the transmission is from $u$ to $v$, we use equation (1) to compute the packet reception rate over links $(u, v)$ and $(v, u)$. Then, based on these values, we compute the expected number of retransmissions. We consider the energy dissipated during the transmission as twice of the energy dissipated during the reception. Some of analytical evaluation parameters have already been presented in the Table I.

\section{B. Analytical results}

Fig.3 and Fig.4 present the maximum energy cost of the node and total energy consumption for the different relay selection schemes. Two different scenarios are studied: the first one with single source node and second one with several source nodes (concurrent flows).

Scenario 1: Fig.3 shows that NFR and NFP are more energy efficient than the remaining other schemes. These results can be explained by the fact that NFR and NFP in some scenarios, use the reliable wireless links (i.e. they are characterized by the least expected number of retransmissions) to route the traffic through several hops. Therefore, the impact of the number of transmissions and receptions at each hop is less important than those based on the most forward, RMP and Angular, which use a longer unreliable wireless links with several number of retransmissions. Note that these results are very similar to those obtained in the section IV, except for RMP scheme.

Scenario 2: The objective of this study is twofold. The first one is to study their energy efficiency. While the second one is to study their behavior with an optimal solution obtained by the linear programming. Fig.4 shows that whatever the number of the source nodes, NFR is better than the remaining other schemes. The results also show that its performance is closer to the optimal solution performance, thanks to the reliable links. By analyzing both the results and Fig.5, we show that 
to minimize the maximum energy consumption, the optimal solution uses disjoint paths ${ }^{3}$ between the source nodes and sink node. Moreover, it forwards its packets in network area where both the node density and expected number of retransmissions are low in order to minimize the energy cost of retransmissions and receptions at each hop and attempts to minimize the path lengths.

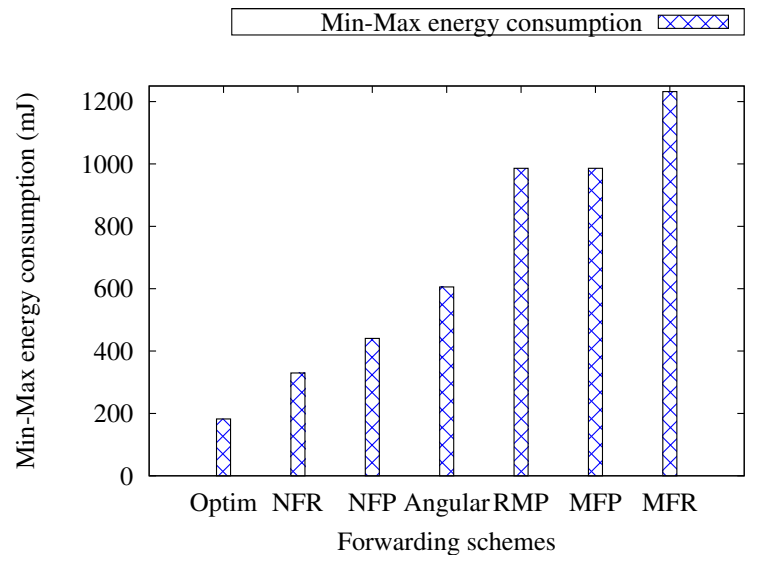

Fig. 3: Maximum energy consumption for each strategy.

\begin{tabular}{|lrr|}
\hline NFP $-\frac{1}{-}$ & Angular $-\square$ & Optimale - \\
MFP $-x$ & NFR $-\square$ & \\
RMP $\longrightarrow$ & MFR - & \\
\hline
\end{tabular}

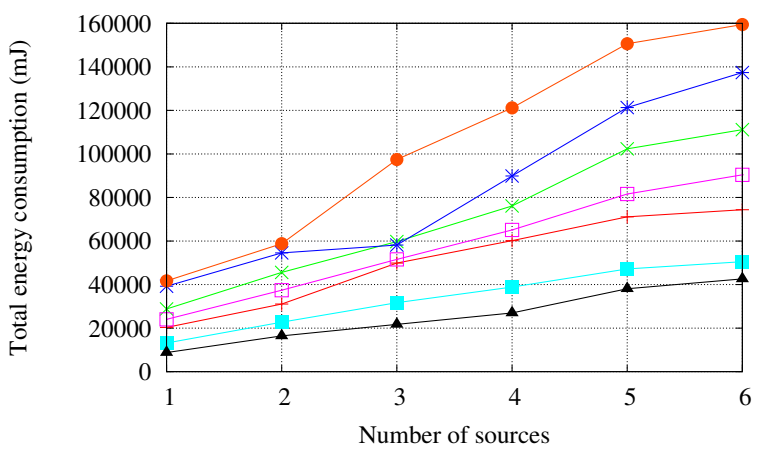

Fig. 4: Total energy consumption for each strategy.

\section{VII. $F F R_{e}$ : FURTHEST FoRWARD WITHIN RELIABLE NEIGHBORS}

Based on the previous concluding remarks about the optimal solution behavior, we propose in this section a new relay selection scheme, $F F R_{e}$, which can outperfom the performance of NFR in terms of the energy efficiency and number of hops. Unlike the optimal solution approach which does not forward its packets based on the destination position area, $F F R_{e}$ is based on position-based routing and uses the reliable links and attempts to minimize the number of hops between the source nodes and sink node. $F F R_{e}$ classifies the relay

${ }^{3}$ Two paths are disjoint if they have no common relay nodes along the path

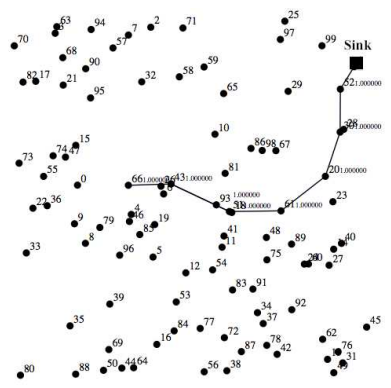

(a) Single flow

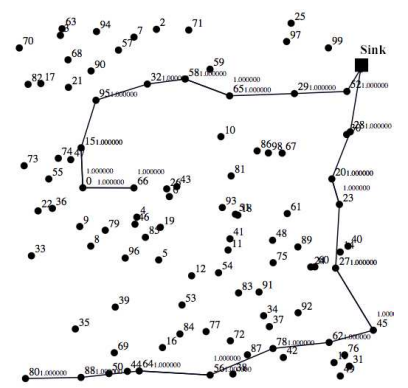

(b) Concurrent flow
Fig. 5: Optimal paths: the linear program adapts the routing paths depending to the number of source nodes.

nodes in the vinicity of the forwarding node into two categories: Reliable neighbors and Unreliable neighbors. Instead of selecting its nearest neighbor as relay node among the reliable neighbors, in $F F R_{e}$, the forwarding node selects the furthest one among these reliable neighbors in order to reduce the number of hops. This can reduce the number of transmissions and receptions along the path. Fig. 6 describes the behavior of $F F R_{e}$ and NFR during their forwarding process.

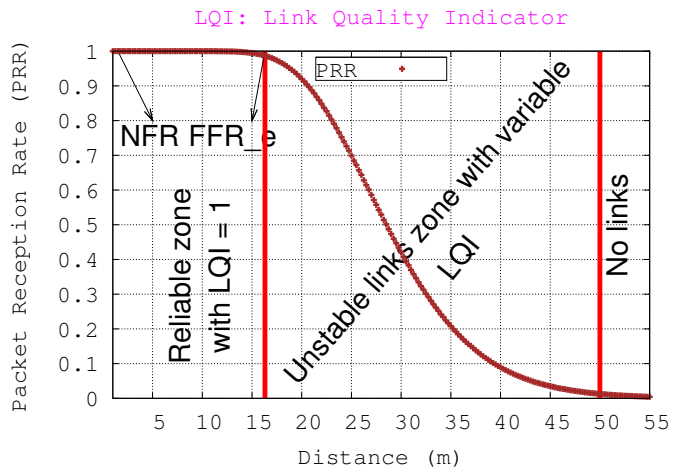

Fig. 6: show where $F F R_{e}$ and NFR select their relay nodes

To highlight the performance of our proposal, we objectively compare its performance with NFR. Fig.7 and Fig.8 display the total energy consumption and average number of hops respectively. Results show the energy efficiency and number of hops efficiency of $F F R_{e}$. They also show that $F F R_{e}$ converges to the optimal solution (see Fig.7).

\section{CONCLUSIONS}

In this paper, we investigate the performance of the different relay selection schemes for contention-based geographic forwarding under realistic radio channel model, where wireless links are unstable and unreliable. In this case, links may present varying link qualities during the time. Through extensive simulations, we first evaluate their performance in terms of packet delivery ratio and duplicated packets. We show that relay selection scheme such as Nearest Forward within Radius $(N F R)$, which selects the relay of each packet in the nearest 


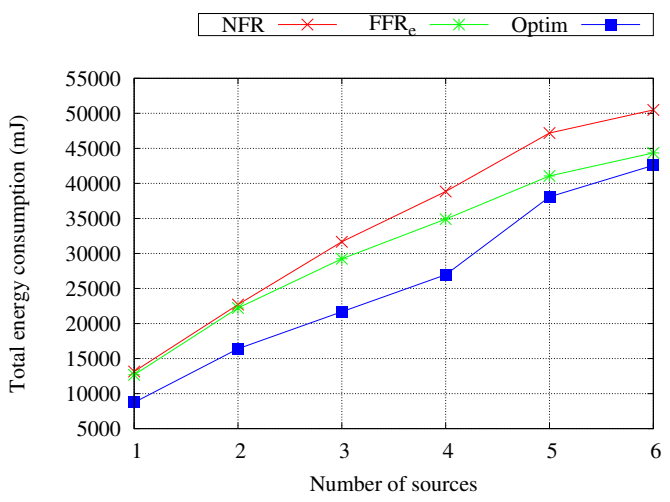

Fig. 7: Total energy consumption

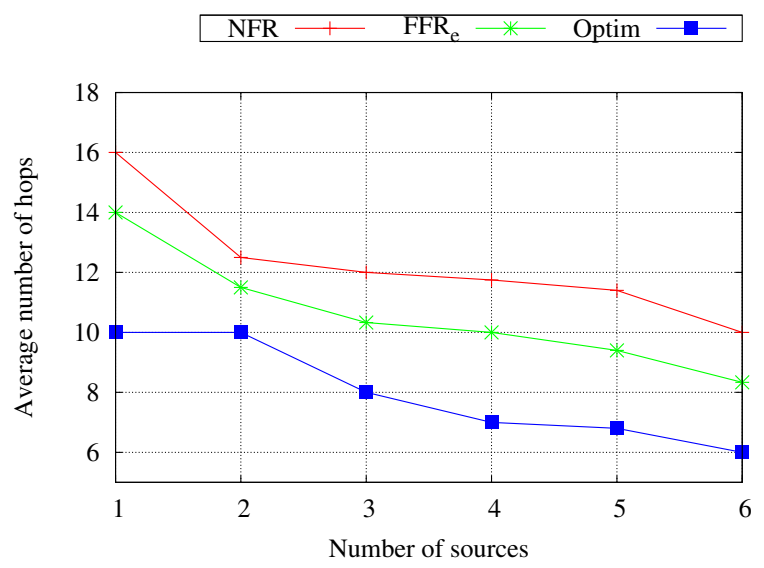

Fig. 8: Average number of hops

neighbors, performs better than the remaining other schemes. Then, we extend the previous work in multi-hop wireless network by analytically evaluate their energy efficiency. A linear programming was developed to calculate an optimal path which minimizes the maximum node cost and the total energy consumption. Based on these results, we also show that NFR outperforms the remaining other schemes. We propose the Furthest Forward within Reliable neighbors $\left(F F R_{e}\right)$, which attempts to minimize both the expected number of transmissions per-hop and number of hops. The performance evaluation shows that $F F R_{e}$ allows to save more energy than NFR by minimizing the number of hops from the source nodes and the destination node.

\section{REFERENCES}

[1] Wsnet's web site. http://wsnet.gforge.inria.fr/

[2] S. Son B. Blum, T. He and J. Stankovic. Igf: A state-free robust communication protocol for wireless sensor networks. Technical report, Tech. Report CS-2003-11, Dept. of Computer Science University of Virginia Charlottesville, VA 22904, April 2003.

[3] Dazhi Chen and Pramod K. Varshney. A survey of void handling techniques for geographic routing in wireless networks. IEEE Communications Surveys and Tutorials, 9(1-4):50-67, 2007.

[4] Douglas S. J. De Couto, Daniel Aguayo, John Bicket, and Robert Morris. A high-throughput path metric for multi-hop wireless routing. In Proceedings of the 9th annual international conference on Mobile computing and networking, MobiCom '03, pages 134-146, San Diego, CA, USA, 14-19 September 2003.

[5] R. Fourer, D. M. Gay, and B. W. Kernighan. A modeling language for mathematical programming. Management Science, 36(5):519-554, 1990.

[6] Marc Heissenbüttel, Torsten Braun, Thomas Bernoulli, and Markus Walchli. Blr: Beacon-less routing algorithm for mobile ad-hoc networks. Elsevier's Computer Communications Journal (Special Issue, 27:10761086, 2004.

[7] Ting-Chao Hou and Victor Li. Transmission range control in multihop packet radio networks. IEEE Transactions on Communications, 34(1):38-44, 1986.

[8] Ilog, Inc. Solver cplex. http://www.ilog.fr/products/cplex/, 2003.

[9] Xu Jiu-qiang, Sun Pei-gang, Zhao Hai, Zhang Xi-yuan, Zhu Jian, and Cheng Da-wei. An analytical model for link evaluation of wireless sensor networks. In Proceedings of the 2007 IFIP International Conference on Network and Parallel Computing Workshops, NPC '07, pages 386-391, Dalian, China, 18-21 September 2007.

[10] A. Helmy K. Seada, M. Zuniga and B. Krishnamachari. Energy-efficient forwarding strategies for geographic routing in lossy wireless sensor networks. In In Proceedings of the 2nd international conference on Embedded networked sensor systems (SenSys 2004), pages 108-121, 35 November 2004.

[11] Brad Karp and H. T. Kung. Gpsr: Greedy perimeter stateless routing for wireless networks. In Proceedings of the 6th annual international conference on Mobile computing and networking, MobiCom '00, pages 243-254, Boston, MA, USA., 6 - 11 August 2000.

[12] Evangelos Kranakis, School Of Computer Science, Harvinder Singh, and Jorge Urrutia. Compass routing on geometric networks. In in Proc. 11 th Canadian Conference on Computational Geometry, pages 51-54 Vancouver, British Columbia, Canada, August 1999.

[13] Randolph Nelson and Leonard Kleinrock. The spatial capacity of a slotted aloha multihop packet radio network with capture. IEEE Transactions on Communications, 32(6):684-694, 1984

[14] Vijay Raghunathan, Curt Schurgers, Sung Park, Mani Srivastava, and Barclay Shaw. Energy-aware wireless microsensor networks. IEEE Signal Processing Magazine, 19(2):40-50, 2002.

[15] Hideaki Takagi and Leonard Kleinrock. Optimal transmission ranges for randomly distributed packet radio terminals. IEEE Transactions on Communications, 32(3):246-257, 1984.

[16] Alec Woo, Terence Tong, and David Culler. Taming the underlying challenges of reliable multihop routing in sensor networks. In Proceedings of the 1st international conference on Embedded networked sensor systems, SenSys '03, pages 14-27, Los Angeles, California, USA, November 2003.

[17] Yuyan Xue, Mehmet C. Vuran, and Byrav Ramamurthy. Cost efficiency of anycast-based forwarding in duty-cycled wsns with lossy channel. In Proceedings of the Seventh Annual IEEE Communications Society Conference on Sensor, Mesh and Ad Hoc Communications and Networks, SECON 2010, pages 520-528, Boston, Massachusetts, USA, 21-25 June 2010.

[18] Marco Zúñiga Zamalloa and Bhaskar Krishnamachari. An analysis of unreliability and asymmetry in low-power wireless links. ACM Transactions on Sensor Networks (TOSN), 3(2), 2007. 